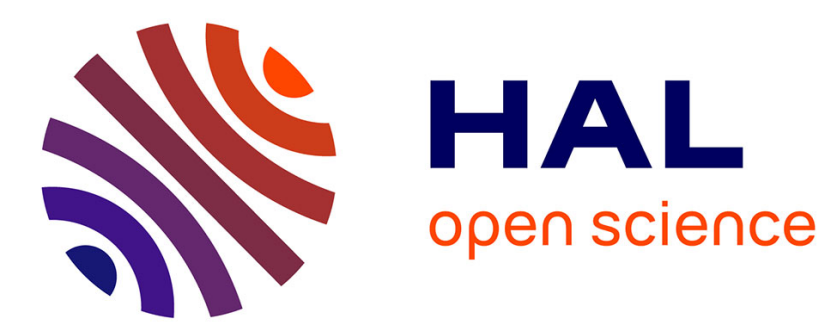

\title{
Sporadic late-onset nemaline myopathy with monoclonal gammopathy of undetermined significance
}

\author{
Akinori Uruha, Olivier Benveniste
}

\section{To cite this version:}

Akinori Uruha, Olivier Benveniste. Sporadic late-onset nemaline myopathy with monoclonal gammopathy of undetermined significance. Current Opinion in Neurology, 2017, 30 (5), pp.457 - 463. 10.1097/WCO.0000000000000477 . hal-01684027

\section{HAL Id: hal-01684027 \\ https://hal.sorbonne-universite.fr/hal-01684027}

Submitted on 15 Jan 2018

HAL is a multi-disciplinary open access archive for the deposit and dissemination of scientific research documents, whether they are published or not. The documents may come from teaching and research institutions in France or abroad, or from public or private research centers.
L'archive ouverte pluridisciplinaire $\mathbf{H A L}$, est destinée au dépôt et à la diffusion de documents scientifiques de niveau recherche, publiés ou non, émanant des établissements d'enseignement et de recherche français ou étrangers, des laboratoires publics ou privés. 
Sporadic late-onset nemaline myopathy with monoclonal gammopathy of undetermined significance

Akinori Uruha and Olivier Benveniste

Akinori Uruha, MD, PhD

Université Pierre et Marie Curie, Paris VI (UPMC), Institut National de la Santé et de la Recherche Médicale (INSERM), Unité Mixte de Recherche (UMR) 974, Centre de Recherche en Myologie, Institut de Myologie, Groupe Hospitalier Pitié-Salpêtrière (GHPS), Paris, France

Olivier Benveniste, $\mathrm{MD}, \mathrm{PhD}$

Département de Médecine Interne et Immunologie Clinique, UPMC, Assistance Publique Hôpitaux de Paris, GHPS, INSERM UMR974, DHU I2B, and Centre de Référence des Pathologies Neuromusculaires Paris Est, Institut de Myologie, Paris, France

Correspondence to Akinori Uruha. 105, boulevard de l'Hôpital, 75013 Paris Cedex 13, France. Tel : +33 140779684; Fax : +33 140778129; E-mail: a.uruha@gmail.com 


\begin{abstract}
Purpose of review Sporadic late-onset nemaline myopathy (SLONM) with monoclonal gammopathy of undetermined significance (MGUS) is a rare subacute progressive muscle disease. The prognosis is poor due to severe respiratory insufficiency. Recently, however, autologous stem cell transplantation following high-dose melphalan (HDM-SCT) has been shown to be effective unless there is delay before the treatment. Therefore, early recognition of the disease is important. This review gives an overview of recent advances in SLONM-MGUS, which could help to understand clinical and pathological features and treatment.

Recent findings $\quad$ Efficacy of HDM-SCT has been demonstrated in a long-term observation study. Subsequently, reports from other groups also have supported it. Furthermore, efficacy of chemotherapy toward plasma cell dyscrasia without SCT have been reported as well. A few case reports have suggested the presence of cardiac involvement related to SLONM-MGUS. Summary SLONM-MGUS is now considered as a treatable disease. Anti-plasma cell dyscrasia therapy is a promising therapeutic option. Meanwhile, the pathomechanic link between muscle degeneration and monoclonal gammopathy remains unclear, and further investigations are warranted.
\end{abstract}




\section{Keywords}

Head drop

Nemaline body

Monoclonal gammopathy of undetermined significance (MGUS)

Autologous stem cell transplantation following high-dose melphalan

\section{Key points}

- Sporadic late-onset nemaline myopathy (SLONM) with monoclonal gammopathy of undetermined significance (MGUS) shows subacute progressive muscle weakness with severe respiratory insufficiency.

- Head drop, MGUS and nemaline body are typical signs of SLONM-MGUS.

- Heart failure possibly appears as an extramuscular involvement.

- Anti-plasma cell dyscrasia therapy including autologous stem cell transplantation following high-dose melphalan is effective. 


\section{Introduction}

In 1963, Shy, et al. originally reported a form of congenital myopathy characterized by the unique cytoplasmic aggregates, which looked like coils of thread or rods, in muscle cells [1]. The aggregate was called as nemaline body (NB) after "nema" meaning thread in Greek, and the disease was named as nemaline myopathy. Currently, nemaline myopathy is regarded as a heterogeneous disease entity. Most of the cases show child-onset and usually chronic progressive course with autosomal recessive or dominant inheritance pattern, and now at least 11 causative genes are identified: TPM3, NEB, ACTA1, TPM2, TNNT1, KBTBD13, CFN2, KLHL40, KLHL41, LMOD3, and MYPN [2]. Meanwhile, adult-onset form is also occasionally seen. Some of such patients have actually congenital nemaline myopathy, the symptom of which develops insidiously and manifests obviously in the adulthood. Other patients show literally adult-onset and subacute progression without any evidence of inheritance. This form is called as sporadic late-onset nemaline myopathy (SLONM), which was first described by Engel in 1966 [3]. However, yet, SLONM is multifactorial. Concomitant monoclonal gammopathy of undetermined significance (MGUS) or multiple myeloma, and human immunodeficiency virus (HIV) infection have been reported in SLONM patients [4-30]. SLONM with HIV infection and that with neither MGUS or HIV appear to be heterogeneous, although SLONM with HIV infection is usually responsive to corticosteroid, intravenous immunoglobulin (IVIg), and plasmapheresis [24, 25, 27, 30]. On the other hand, SLONM with MGUS (SLONM-MGUS) patients show a particular phenotype: severe weakness and atrophy in axial, limb-girdle, and occasionally distal muscles, dysphagia, and respiratory insufficiency, which are often refractory to immunotherapy [4-22]. Of note, respiratory involvement is lethal, and the majority of the patients died within 1 to 5 years from the onset [8]. Because of this poor prognosis, our group has shown that autologous stem cell transplantation following high-dose melphalan (HDM-SCT) can lead to dramatical improvement in the SLONMMGUS patients $[10,11,16]$. Subsequently, the efficacy of HDM-SCT or chemotherapy to the plasma cell dyscrasia has been demonstrated by other groups as well [13, 15, 17-19]. Hence, today, 
regardless of rarity of the disease, the significance of early recognition of SLONM-MGUS is increasing since the disease is lethal but now can be considered treatable. This review gives an overview of clinical and pathological features, treatments, and recent advances of SLONM-MGUS with some discussion of the etiology. 


\section{Clinical features}

Through our literature search in PubMed until April 2017, we identified 31 SLONMMGUS patients in 19 articles [4-22]. On review of the cases, the disease usually appears in their late 30 s or older (the median of onset age is 47 years old [range: 27 - 78]). Elderly onset (65 years of age or older) is reported in 2 patients [8]. Sex ratio is $1.6: 1$ (19 males versus 12 females). Muscle weakness and atrophy are observed mainly in axial and limb-girdle muscles. Distal and facial muscle weakness, dysphagia, dysarthria, and muscle pain can also be seen in some patients. Head drop is characteristically seen. Head drop or neck muscle weakness is described in more than half of the patients during their clinical course (head drop: 12 patients; neck muscle weakness: 6 patients). Symptoms in neck muscles, including back neck muscle pain, are recorded as an initial manifestation in 9 patients. Head drop or severe neck extensor muscle weakness appearing in the early (ambulant) stage of disease is well known in amyotrophic lateral sclerosis, myasthenia gravis, and several other neuromuscular diseases, but it is infrequent even in those diseases. The relatively frequent occurrence of head drop in SLONM-MGUS may show that it is a clinical feature suggestive of SLONM-MGUS, as Lomen-Hoerth, et al. also advocated [31]. Severe respiratory involvement is also characteristic in SLONM-MGUS. In the report of Chahin, et al., 5 out of 7 patients with MGUS died of respiratory failure within 1 to 5 years if they had no treatment or ineffective therapy [8].

A recent series of case reports have suggested a possibility of cardiac muscle involvement in SLONM-MGUS $[19,20,22]$. In the report of Belhomme, et al., one SLONM-MGUS patient was admitted to the intensive care unit due to acute heart failure after 2 years from the onset of muscle weakness at the age of 55 years [22]. The left ventricular ejection fraction (LVEF) was reduced to $30 \%$, but it recovered to $60 \%$ after chemotherapy of bortezomib, cyclophosphamide and dexamethasone, together with selective beta-blockers. In another report, a 37-year-old patient was pointed out to have left ventricular systolic dysfunction due to diffuse hypokinesia within 2 years after the onset of muscle weakness [20]. Subsequently, severe congestive heart failure with 
$20 \%$ of LVEF appeared, and three cardiac arrests due to ventricular tachycardia and fibrillation occurred, requiring intensive care and an implantable cardioverter defibrillator. Although response to anti-plasma cell dyscrasia therapy is not mentioned, the report has described that his heart condition was controlled by ivabradine, a sinus node $\mathrm{I}_{\mathrm{f}}$ channel inhibitor, with the rehabilitation program. In the other article, an asymptomatic left ventricular systolic dysfunction with a moderate reduction of LVEF to $42 \%$ was detected in a 44 -year-old patient 5 years after the onset of muscle weakness, although response to the HDM-SCT is not mentioned [19]. These 3 cases showing cardiac abnormality developed 2-5 years after the onset of SLONM-MGUS may imply the presence of cardiomyopathy as an extramuscular manifestation. The case with improvement after anti-plasma cell dyscrasia therapy is especially suggestive. However, only anecdotal evidence from a limited number of patients is available at present. Also, there has been no pathological evidence, such as NB in cardiac muscle cells, yet. Further investigation is necessary to solidly demonstrate cardiac muscle involvement in SLONM-MGUS.

As a concurrent autoimmune disorder, vitiligo that developed 2-year prior to the onset was reported in one patient, although the actual association remains unknown [14]. The patient showed significant improvement in muscle strength by monthly IVIg, although the response of immunotherapy toward the skin lesions was not mentioned.

Serum creatine kinase levels are within, or occasionally lower than, normal range in almost all patients. About MGUS, both types of free light chain predominance $(\kappa$ and $\lambda)$ are described (17: 12 . Two different M proteins, IgG- $\kappa$ and $-\lambda$, were simultaneously present in one patient [biclonal gammopathy] [12]. Not available in one patient). Correlation of the predominant light chain type and serum M protein levels with clinical course and severity is not well known. However, the re-increase of the serum M protein levels after HDM-SCT appears to be associated with the relapse [16]. One patient was described as having multiple myeloma, not MGUS, as her bone marrow aspiration showed clonal plasma cells occupied $20 \%$ of the cells [18]. 
Screening for monoclonal immunoglobulin had been performed conventionally by means of protein electrophoresis and immunofixation. However, in the early 2000 s, the serum free light chain (FLC) assay was developed $[32,33]$. This technology is based on immunonephelometry using specific antibodies that react with the epitopes which are hidden when bound to a heavy chain but exposed when not associated with it $[32,33]$. Some studies demonstrated that serum FLC assay in combination with serum protein electrophoresis and immunofixation could detect light chains more sensitively than the respective assay alone. Therefore, currently, the combination of the assays is recommended for screening of monoclonal gammopathy [33, 34]. When a patient is suspected to have SLONM, measurement with the combination of the assays may lead to avoid overlooking MGUS.

In muscle magnetic resonance images, patchy high intensity on T2-weighted images or short-tau inversion recovery images, which can reflect edematous change and is often observed in inflammatory myopathies, was reported, although only few reports described the images precisely $[14,21]$.

Electromyography shows myopathic changes with abnormal spontaneous activity at rest in almost all cases when the results of the test were described. This pattern is observed commonly in inflammatory myopathies, albeit not disease-specific [35]. 


\section{Muscle pathology}

The main pathologic feature is NB in myofibers [Figure 1a]. The size of NB is smaller than $1 \mu \mathrm{m}[8]$ and than NB seen in congenital nemaline myopathy; NB in SLONM-MGUS looks like "sand" rather than nema (thread) on light microscopy. NB often occupy the whole sections of atrophic fibers. When NB are seen in myofibers with larger diameter, they are accumulated in the central portion. Proportion of myofibers bearing NB is variable among patients and among different muscle samples of the same patient in whom more than one muscles were biopsied [8]. Repeated muscle biopsy or re-assessment is sometimes required to detect NB [12, 14-16, 21, 22]. To observe NB, modified Gomori trichrome stain is useful. NB are visualized as fine cytoplasmic aggregates with dark to reddish purple color on the staining. On hematoxylin and eosin stain, NB seem mere basophilic areas and will be difficult to detect $[8,11,12,15,22]$. Observation of trichromatically stained frozen sections with higher magnification is recommended. NB are positively stained on immunohistochemistry for Z-band proteins such as alpha-actinin and myotilin because NB are derived from degenerated $\mathrm{Z}$ bands. On electron microscopy, NB are morphologically confirmed by the high electron density similar to $\mathrm{Z}$ bands and the presence of internal lattice-like structure [Figure 1b, c]. The ultrastructural observation contributes to distinguish from other cytoplasmic aggregates such as cytoplasmic body and spheroid body. Intranuclear rods, which are known to be associated with severe-infantile form of nemaline myopathy with $A C T A 1$ mutation and relatively mild form with $M Y P N$ mutation, has been rarely reported $[2,4,8,36]$. It should be noted that NB per se is not specific although its presence is a mandatory finding of nemaline myopathies. NB can be observed also in idiopathic inflammatory myopathies, alcoholic myopathy, advanced stage of muscular dystrophies, hypothyroidism, cancer invasion, and chronic neuropathy like spinal muscular atrophy [37-42]. Empirically, a small number of fibers with NB can be seen physiologically if the muscle is biopsied near tendon.

In congenital nemaline myopathy, bimodal distribution of fiber size, type 1 fiber predominance and atrophy, and type 2B fiber deficiency are observed. Also in SLONM-MGUS, 
type 1 fiber predominance and / or atrophy was reported in some cases, requiring attention in terms of differential diagnosis from adult-onset congenital nemaline myopathy [5-8, 20, 21]. Although there is limited data on type 2B fiber deficiency, lack of type 2B fiber deficiency may be a pathologic differential point to distinguish both conditions [21].

Necrotic or regenerating fibers are not seen, or rare if present, unlike muscular dystrophy and immune-mediated necrotizing myopathy. There is no significant endomysial lymphocyte infiltration as seen in polymyositis and inclusion body myositis. Expression of major histocompatibility complex (MHC) class I is not observed, or detected only on sarcolemma, immunohistochemically. Chahin, et al. mentioned that, in their experience, the biopsy findings of SLONM-MGUS were similar to HIV-associated nemaline myopathy [8].

Immunoreactivity for light chains on muscle tissues, mainly sarcolemma, was reported in 4 patients, while amyloid deposition was not observed $[6,7,15,16]$ Based on the facts, Doppler, et al. inferred that SLONM-MGUS was a continuum of light-chain deposition disease (LCDD). LCDD is a rare disease characterized by deposition of monoclonal immunoglobulin light chains (commonly $\kappa$ type) on multiple organs (mainly kidney, liver, and heart) [43-46]. The deposits are not stained with Congo-red stain and do not exhibit a fibrillar structure like amyloid but a fine granular appearance on electron microscopy $[47,48]$. Their thought is consistent with the fact that light chain deposition was observed on skeletal muscles. However, the difference of affected organs / tissues between LCDD and SLONM-MGUS will have to be considered; LCDD is a multiple organ disease, but, in contrast, SLONM-MGUS affects exclusively muscles (skeletal and possibly cardiac). This selectivity may rather imply the implication of autoimmunity in the pathogenesis. 


\section{Treatment}

During the decade, our group has shown the efficacy of HDM-SCT in SLONM-MGUS

[Figure 2] [10, 11, 16]. HDM-SCT has been established as the treatment for plasma cell dyscrasia including amyloid light chain (AL) amyloidosis and POEMS syndrome (Polyneuropathy, Organomegaly, Endocrinopathy / edema, M protein, Skin abnormalities; Crow-Fukase syndrome). Considering the similarity to the etiology of SLONM-MGUS and its severity and rapid progression, HDM-SCT was tried [10, 11]. Since the initial reports in 2008, there have been reports of 11 patients receiving HDM-SCT from several different groups [10, 11, 13, 15-17, 19$]$. Favorable response is observed in 10 out of the 11 patients. Long-standing improvement in muscle strength were observed in most of the patients, although second HDM-SCT and additional antiplasma cell chemotherapy (lenalidomide, bortezomib, cyclophosphamide, and dexamethasone) were added in 5 patients $[15,16]$. The increase of the serum M protein levels preceded clinical deterioration in the 2 patients who required second HDM-SCT [16]. Monitoring of M protein levels may be helpful for follow-up of patients. Disappearance of NB were confirmed when muscle biopsy was performed after the treatment $[10,11]$. Age at onset of muscle weakness, severity of muscle weakness before HDM-SCT, the level and type of M protein, and results of bone marrow aspiration before the transplantation did not correlate with the clinical response [16]. One patient died of Salmonella typhi septicemia 5 months after the treatment, despite significant improvement in muscle strength [19]. The only one patient who showed neither a clinical nor hematologic response to the HDM-SCT received additional chemotherapy, but later she died [16]. The factors of unfavorable outcome in the patient were considered as a long disease course before HDM-SCT (11 years) and a poor hematologic response to the treatments.

The mortality rates within 100 days after HDM-SCT were reported to be $1.9 \%$ in multiple myeloma and $13-24 \%$ in AL amyloidosis $[49,50]$. The mortality might be influenced by involved organs. A large population study, enrolling 701 consecutive patients with AL amyloidosis, revealed that the survival of the participants treated by HDM-SCT was adversely affected by the presence 
of cardiac involvement [50]. The same study also reported that there was no difference in the survival of the participants aged over 65 years compared to younger participants (eligible patients up to age 80 years were treated in the study) [50], although a prior study of another group had suggested that patients 65 years of age or older should not be considered for the treatment [51]. In SLONM-MGUS, as described early, cardiac muscle involvement is possible, and elderly patients can also be encountered [8, 19, 20, 22]. In such cases, indication of HDM-SCT should be considered more carefully. Recently, some chemotherapies without SCT for multiple myeloma have been developed [52]. In patients with risk, such therapies might be an option. Belhomme, et al. reported that a patient with cardiac failure possibly related to MGUS received a chemotherapy without SCT (four cycle of the bortezomib-cyclophosphamide-dexamethasone) and showed a significant improvement in motricity and cardiac function [22]. Montagnese, et al. also described a patient with one cycle of lenalidomide-dexamethasone therapy showed a significant improvement [18]. Furthermore, in the report of Doppler, et al., bortezomib with dexamethasone \pm cyclophosphamide was effective toward relapse after HDM-SCT [15]. These cases suggest a possible efficacy of anti-plasma cell dyscrasia therapy without SCT in SLONM-MGUS.

Immunotherapy and plasmapheresis without or before any anti-plasma cell dyscrasia therapy were done in 17 patients $[4-8,11,12,14,16,17,21,22]$. The outcomes are summarized in Table. Among immunotherapies, IVIg alone or in a combination with other immunotherapies seems relatively hopeful; it showed favorable responses in 6 out of 9 patients (significant improvement in $3[14,21]$, modest improvement in 1 [12], and inhibition of progression in 2 [8, 21]). There is one report showing a patient whose muscle strength improved and remained stable with methylprednisolone and azathioprine [7], but, collectively, corticosteroid alone or with immunosuppressants would not be promising $[4-6,8,11,12,21]$.

Considering the increase of serum $\mathrm{M}$ protein levels preceding the relapse after HDMSCT, SLONM-MGUS may be classified as a disease associated with toxic M proteins, like POEMS syndrome [16]. In POEMS syndrome, not the deposition of the monoclonal 
immunoglobulins in affected tissues but the antibody activity toward autoantigens, which is possibly augmented by other humoral mediators such as VEGF, is considered causative [53]. POEMS syndrome and other related disorders show a similar response to HDM-SCT as observed in the SLONM-MGUS patients. Yet, the exact role of the M proteins in SLONM-MGUS remains unknown. Albeit lack of consistency of efficacy, the presence of patients showing favorable responses to immunotherapies including IVIg may provide indirect evidence that SLONM-MGUS has a dysimmune etiology. 


\section{Conclusion}

SLONM-MGUS is a severe, lethal disease, but today it is considered as a treatable disease. There are 3 typical features of SLONM-MGUS in clinical, serological, and pathological aspects: head drop, MGUS, and NB. Although each of them is not a specific finding, their combination is a hallmark of the disease. Anti-plasma cell dyscrasia therapy such as HDM-SCT is beneficial for SLONM-MGUS patients.

At present, the pathomechanic link between muscle degeneration and monoclonal gammopathy remains unclear. Further investigation is necessary and the better understanding of the pathogenesis will lead to development of more effective and safer therapy. 
Financial support and sponsorship The current work was not supported by any funding

Conflicts of interest $\quad$ A. Uruha reports grants from The Uehara Memorial Foundation and from Japan Foundation for Neuroscience and Mental Health.

Figure Legend

Figure 1 (a) Nemaline bodies are observed as fine and reddish purple aggregates in cytoplasm on modified Gomori trichrome stain. Frozen biopsied skeletal muscle of a SLONMMGUS patient. (b) On electron microscopy, nemaline bodies show high electron density. (c) The structure is lattice-like. Longitudinal section.

Figure $2(\mathrm{a}, \mathrm{b})$ The SLONM-MGUS patient could not raise her arms above her shoulders or walk without assistance before treatment of HDM-SCT. (c, d) After the treatment, the patient could raise her arms above her arms and walk independently. 


\section{References and recommended reading}

1 Shy GM, Engel WK, Somers JE, et al. Nemaline myopathy. A new congenital myopathy. Brain 1963; 86: 793-810.

2 Miyatake S, Mitsuhashi S, Hayashi YK, et al. Biallelic mutations in MYPN, encoding myopalladin, are associated with childhood-onset, slowly progressive nemaline myopathy. Am J Hum Genet 2017; 100: 169-178.

Engel AG. Late-onset rod myopathy (a new syndrome?): light and electron microscopic observations in two cases. Mayo Clin Proc 1966; 41: 713-741.

4 Engel WK, Oberc MA. Abundant nuclear rods in adult-onset rod disease. J Neuropathol Exp Neurol 1975; 34: 119-132.

Seitz RJ, Toyka KV, Wechsler W. Adult-onset mixed myopathy with nemaline rods, minicores, and central cores: a muscle disorder mimicking polymyositis. J Neurol 1984; 231: 103-108.

6 Eymard B, Brouet JC, Collin H, et al. Late-onset rod myopathy associated with monoclonal gammopathy. Neuromuscul Disord 1993; 3: 557-560.

7 Deconinck N, Laterre EC, Van den Bergh PY. Adult-onset nemaline myopathy and monoclonal gammopathy: a case report. Acta Neurol Belg 2000; 100: 34-40.

$8 \quad * *$ Chahin N, Selcen D, Engel AG. Sporadic late onset nemaline myopathy. Neurology 2005; 65: 1158-1164.

The authors have delineated clinical and pathological features of SLONM patients.

9 Keller CE, Hays AP, Rowland LP, et al. Adult-onset nemaline myopathy and monoclonal gammopathy. Arch Neurol 2006; 63: 132-134.

10 Benveniste O, Laforet P, Dubourg O, et al. Stem cell transplantation in a patient with lateonset nemaline myopathy and gammopathy. Neurology 2008; 71: 531-532.

11 Voermans NC, Minnema M, Lammens M, et al. Sporadic late-onset nemaline myopathy effectively treated by melphalan and stem cell transplant. Neurology 2008; 71: 532-534. 
12 Irodenko VS, Lee HS, de Armond SJ, et al. Adult nemaline myopathy with trabecular muscle fibers. Muscle Nerve 2009; 39: 871-875.

13 Novy J, Rosselet A, Spertini O, et al. Muscle Nerve 2010; 41: 286-287.

14 Milone M, Katz A, Amato AA, et al. Sporadic late onset nemaline myopathy responsive to IVIg and immunotherapy. Muscle Nerve 2010; 41: 272-276.

15 Doppler K, Knop S, Einsele H, et al. Sporadic late-onset nemaline myopathy and immunoglobulin deposition disease. Muscle Nerve 2013; 48: 983-988.

$16 * *$ Voermans NC, Benveniste O, Minnema MC, et al. Sporadic late-onset nemaline myopathy with MGUS: long-term follow-up after melphalan and SCT. Neurology 2014; 83: 2133-2139.

We have shown efficacy of HDM-SCT for SLONM-MGUS patients in a long-term observation.

17 Maeda MH, Ohta H, Izutsu K, et al. Sporadic late-onset nemaline myopathy as a rare cause of slowly progressive muscle weakness with young adult onset. Muscle Nerve 2015; 51: 772-774.

$18 *$ Montagnese F, Portaro S, Musumeci O, et al. Sporadic late-onset nemaline myopathy in a woman with multiple myeloma successfully treated with lenalidomide/dexamethasone. Muscle Nerve 2015; 51: 934-935.

This article has shown the efficacy of chemotherapy for plasma cell dyscrasia in SLONMMGUS. Also, particularly, the patient developed muscular symptom after SCT.

19 Belkhribchia MR, Tazi I, Louhab N, et al. Autologous stem cell transplantation in a patient with sporadic late-onset nemaline myopathy and monoclonal gammopathy: First Moroccan experience. Presse Med 2017; 46: 122-125.

20 Sarullo FM, Vitale G, Di Franco A, et al. Nemaline myopathy and heart failure: role of ivabradine; a case report. BMC Cardiovasc Disord 2015; 15: 5. 
21 Mizuno Y, Mori-Yoshimura M, Okamoto T, et al. Two cases of sporadic late onset nemaline myopathy effectively treated with immunotherapy. Rinsho Shinkeigaku 2016; 56: 605-611.

$22 *$ Belhomme N, Maamar A, Le Gallou T, et al. Rare myopathy associated to MGUS, causing heart failure and responding to chemotherapy. Ann Hematol 2017; 96: 695-696. This article has shown the efficacy of targeted chemotherapy for plasma cell dyscrasia without HDM-SCT in SLONM-MGUS. Also, a possibility of cardiac involvement in SLONM-MGUS has been shown. The cardiac function significantly improved after the treatment.

23 Dalakas MC, Pezeshkpour GH, Flaherty M. Progressive nemaline (rod) myopathy associated with HIV infection. N Engl J Med 1987; 317: 1602-1603.

24 Gonzales MF, Olney RK, So YT, et al. Subacute structural myopathy associated with human immunodeficiency virus infection. Arch Neurol 1988; 45: 585-587.

25 Simpson DM, Bender AN. Human immunodeficiency virus-associated myopathy: analysis of 11 patients. Ann Neurol 1988; 24: 79-84.

26 Cabello A, Martinez-Martin P, Gutierrez-Rivas E, Madero S. Myopathy with nemaline structures associated with HIV infection. J Neurol 1990; 237: 64-66.

27 Dwyer BA, Mayer RF, Lee SC. Progressive nemaline (rod) myopathy as a presentation of human immunodeficiency virus infection. Arch Neurol 1992; 49: 440.

28 Maytal J, Horowitz S, Lipper S, Poiesz B, Wang CY, Siegal FP. Progressive nemaline rod myopathy in a woman coinfected with HIV-1 and HTLV-2. Mt Sinai J Med 1993; 60: 242-246.

29 Feinberg DM, Spiro AJ, Weidenheim KM. Distinct light microscopic changes in human immunodeficiency virus-associated nemaline myopathy. Neurology 1998; 50: 529-531.

30 Madonia P, Wilson J, Bican O, et al. HIV, rods, and the muscles - a discussion about HIV-associated nemaline rod myopathy. J La State Med Soc 2012; 164: 320-323. 
31 Lomen-Hoerth C, Simmons ML, Dearmond SJ, et al. Adult-onset nemaline myopathy: Another cause of dropped head. Muscle Nerve 1999; 22: 1146-1150.

32 Bradwell AR, Carr-Smith HD, Mead GP, et al. Highly sensitive, automated immunoassay for immunoglobulin free light chains in serum and urine. Clin Chem 2001; 47: 673-680.

33 Dispenzieri A, Kyle R, Merlini G, et al. International Myeloma Working Group guidelines for serum-free light chain analysis in multiple myeloma and related disorders. Leukemia 2009; 23: 215-224.

34 Katzmann JA, Dispenzieri A, Kyle RA, et al. Elimination of the need for urine studies in the screening algorithm for monoclonal gammopathies by using serum immunofixation and free light chain assays. Mayo Clin Proc 2006; 81: 1575-1578.

35 Kimura J: Electrodiagnosis in Diseases of Nerve and Muscle: Principles and Practice. Edition 3. Oxford University Press. 2001; 797-802.

36 Ryan MM, Ilkovski B, Strickland CD, et al. Clinical course correlates poorly with muscle pathology in nemaline myopathy. Neurology 2003; 60: 665-673.

37 Cape CA, Johnson WW, Pitner SE. Nemaline structures in polymyositis. A nonspecific pathological reaction of skeletal muscles. Neurology 1970; 20: 494-502.

38 Martinez AJ, Hooshmand H, Faris AA. Acute alcoholic myopathy. Enzyme histochemistry and electron microscopic findings. J Neurol Sci 1973; 20: 245-252.

39 Reyes MG, Tal A, Abrahamson D, et al. Nemaline myopathy in an adult with primary hypothyroidism. Can J Neurol Sci 1986; 13: 117-119.

40 Konno H, Iwasaki Y, Yamamoto T, et al. Nemaline bodies in spinal progressive muscular atrophy. An autopsy case. Acta Neuropathol 1987; 74: 84-88.

41 Kimura M, Furuta T, Hiruma S, et al. Nemaline bodies of skeletal muscle fibers of the neck in a case of pharyngeal cancer. Pathol Int 1997; 47: 256-259.

42 Sun AP, Ohtsuki Y, Yano T, et al. Typical nemaline bodies presenting in a patient with polymyositis. Med Electron Microsc 2002; 35: 167-172. 
43 Buxbaum JN, Chuba JV, Hellman GC, et al. Monoclonal immunoglobulin deposition disease: light chain and light and heavy chain deposition diseases and their relation to light chain amyloidosis. Clinical features, immunopathology, and molecular analysis. Ann Intern Med 1990; 112: 455-464.

44 Ronco PM, Alyanakian MA, Mougenot B, et al. Light chain deposition disease: a model of glomerulosclerosis defined at the molecular level. J Am Soc Nephrol 2001; 12: 15581565.

45 Ronco P, Plaisier E, Mougenot B, et al. Immunoglobulin light (heavy)-chain deposition disease: from molecular medicine to pathophysiology-driven therapy. Clin J Am Soc Nephrol 2006; 1: 1342-1350.

46 Weichman K, Dember LM, Prokaeva T, et al. Clinical and molecular characteristics of patients with non-amyloid light chain deposition disorders, and outcome following treatment with high-dose melphalan and autologous stem cell transplantation. Bone Marrow Transplant 2006; 38: 339-343.

47 Faa G, Van Eyken P, De Vos R, et al. Light chain deposition disease of the liver associated with AL-type amyloidosis and severe cholestasis: a case report and literature review. J Hepatol 1991; 12: 75-82.

48 Kasahara N, Tamura H, Matsumura O, et al. An autopsy case of light-chain deposition disease. Intern Med 1994; 33: 216-221.

49 Jantunen E, Itälä M, Lehtinen T, et al. Early treatment-related mortality in adult autologous stem cell transplant recipients: a nation-wide survey of 1482 transplanted patients. Eur J Haematol 2006; 76: 245-250.

50 Skinner M, Sanchorawala V, Seldin DC, et al. High-dose melphalan and autologous stemcell transplantation in patients with AL amyloidosis: an 8-year study. Ann Intern Med 2004; 140: 85-93. 
51 Gertz MA, Lacy MQ, Gastineau DA, et al. Blood stem cell transplantation as therapy for primary systemic amyloidosis (AL). Bone Marrow Transplant 2000; 26: 963-969.

52 Sonneveld P, Avet-Loiseau H, Lonial S, et al. Treatment of multiple myeloma with highrisk cytogenetics: a consensus of the International Myeloma Working Group. Blood 2016; 127: 2955-2962.

53 Merlini G, Stone MJ. Dangerous small B-cell clones. Blood 2006; 108: 2520-2530. 
Table. Outcome of therapy.

\begin{tabular}{|c|c|c|c|c|c|c|}
\hline & & Effective & Partially effective & Poor & Total & Reference \\
\hline \multicolumn{2}{|c|}{$\begin{array}{c}\text { HDM-SCT } \\
\pm \text { additional chemotherapy }\end{array}$} & 10 & - & 1 & 11 & $\begin{array}{c}10,11,13,15-17, \\
19\end{array}$ \\
\hline \multirow{5}{*}{$\begin{array}{c}\text { Without / before } \\
\text { HDM-SCT }\end{array}$} & Chemotherapy & 2 & - & - & 2 & 18,22 \\
\hline & IVIg \pm CS / IS & 3 & 2 & 2 & 7 & $\begin{array}{c}8,12,14,17,21, \\
22\end{array}$ \\
\hline & $\begin{array}{l}\text { IVIg + PP } \\
+\mathrm{CS}+\mathrm{IS}\end{array}$ & - & 1 & 1 & 2 & 16,21 \\
\hline & $\mathrm{PP} \pm \mathrm{CS}$ & - & 1 & 3 & 4 & $6,7,16,21$ \\
\hline & $\mathrm{CS} \pm \mathrm{IS}$ & 1 & 2 & 7 & 10 & $4-8,11,12,21$ \\
\hline
\end{tabular}


Effective: significant clinical improvement in muscle strength. Partially effective: slight improvement in muscle strength or no improvement but inhibition of disease progression. Poor: neither improvement nor inhibition of disease progression. HDM-SCT: autologous stem cell transplantation following high-dose melphalan. Chemotherapy: anti-plasma cell dyscrasia agents including lenalidomide and bortezomib, with dexamethasone and cyclophosphamide. IVIg: intravenous immunoglobulin. CS: corticosteroid. IS: immunosuppressant agents. PP:plasmapheresis. 


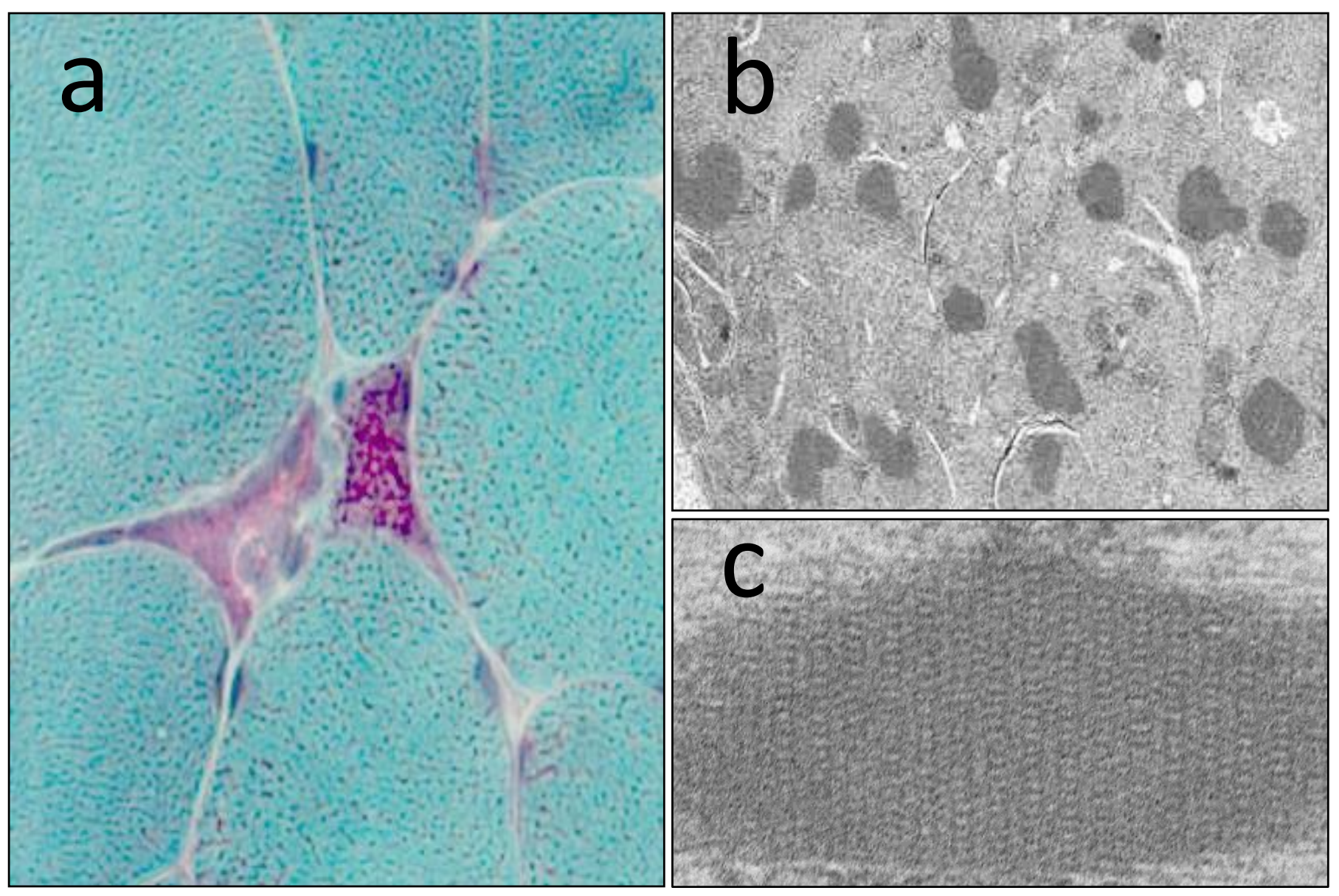


